Pacific

Journal of

Mathematics

LIE SEMIGROUPS WITH TRIPLE DECOMPOSITIONS

Jimmie LAWSON AND Yongdo Lim 


\title{
LIE SEMIGROUPS WITH TRIPLE DECOMPOSITIONS
}

\author{
Jimmie LAWSON AND Yongdo Lim
}

\begin{abstract}
In this paper we establish that the causal order determined by an Ol'shanski semigroup on the corresponding homogeneous space is globally hyperbolic. Using this fact, we present sufficient conditions for a special class of Lie semigroups to admit a canonical "triple decomposition," namely those for which the Lie algebra is of Cayley type. This theory applies in particular to semigroups which are naturally associated to euclidean Jordan algebras as the semigroup of compressions of the symmetric cone of the algebra.
\end{abstract}

\section{Introduction.}

In [18] G.I. Ol'shanski introduced a remarkable class of subsemigroups of Lie groups which have come to be called Ol'shanski semigroups. A typical example of such a semigroup arises in the complexification $G_{\mathbb{C}}$ of a semisimple hermitian Lie group $G$ by taking an $\mathrm{Ad} G$-invariant convex cone $\mathbf{C}$ in the Lie algebra $\mathfrak{g}$ of $G$ and forming the semigroup $S=G \exp (i \mathbf{C})$ in $G_{\mathbb{C}}$. Such semigroups can be viewed as non-commutative analogs of tube domains, and they have played an important role in recent developments both in harmonic analysis and in representation theory (see [11] for survey treatments of many of these new developments).

The existence of (infinitesimally generated) semigroups at the group level manifests itself in the existence of causal structures and causal partial orders at the homogeneous space level [14]. In the harmonic analysis carried out at the homogeneous space level (e.g., in the analysis of kernels in Volterra algebras [5]), it is frequently crucial to know that the partial order is "globally hyperbolic," i.e., that the order intervals are compact. The property of being globally hyperbolic has also played an important role in other contexts, e.g., in the study of partial differential equations and in the causal orders that arise in Lorentzian geometry. Mittenhuber and Neeb have exploited this condition in their study of the exponential function on ordered manifolds with affine connections [17].

In this paper we use recent results of B. Krötz and K.-H. Neeb [13] on hyperbolic cones to prove that the homogeneous causal order arising from an Ol'shanski semigroup is always globally hyperbolic (Theorem 4.4); this 
general result extends earlier work of J. Faraut [5], followed by J. Hilgert and G. Ólafsson [10], who proved it for special cases.

Let $G$ be a Lie group equipped with an involution $\tau$. Then $\tau$ induces an involution on the Lie algebra $\mathfrak{g}$ (making it a symmetric Lie algebra), and $\mathfrak{g}$ is the direct sum of the +1 -eigenspace $\mathfrak{h}$ and the -1 -eigenspace $\mathfrak{q}$. Let $H$ be a $\tau$-fixed subgroup with Lie algebra $\mathfrak{h}$. If $\mathfrak{q}$ contains an $\mathrm{Ad} H$-invariant hyperbolic cone $\mathbf{C}$, then $H(\exp \mathbf{C})$ is an Ol'shanski semigroup. One extremely useful structural property of such semigroups is the existence and uniqueness of the "Ol'shanski polar decomposition": Each element $s$ factors uniquely as $s=h \exp (X), h \in H, X \in \mathbf{C}$. This factorization may be viewed as a semigroup variant of the classical Cartan factorization. In this paper we consider the important special case that the symmetric algebra $\mathfrak{g}$ is of Cayley type (this means that $\mathfrak{q}$ can be written as $A d H$-invariant summands $\mathfrak{q}^{+}+\mathfrak{q}^{-}$, each of which is an abelian subalgebra). In this case we establish that the Ol'shanski semigroup has a unique triple decomposition $S=\exp \left(\mathbf{C}^{-}\right) H \exp \left(\mathbf{C}^{+}\right)$(Theorem 5.3), which may be viewed as a semigroup variant of the Harish-Chandra decomposition. In [15] the first author gave necessary and sufficient conditions for the existence of the Ol'shanski semigroup, given an $\operatorname{Ad}(H)$-invariant hyperbolic cone in $\mathfrak{q}$. A very pleasant feature of the theory established in this paper is that under the mild restriction that the cone is pointed, then for cones of Cayley type the triple decomposition obtains whenever the Ol'shanski semigroup exists.

Semigroups for which the triple decomposition holds include sympletic semigroups, or more generally the conformal compression semigroup of a symmetric cone in an euclidean Jordan algebra. Such semigroups have been studied in detail by K. Koufany in [12]. Relying heavily on Jordan algebra theoretic methods, he established the triple decomposition for this class of semigroups. In the last two sections of this paper we revisit this class of examples and show how these semigroups fit within our framework and how the triple decomposition follows from our general results. We pay particular attention to the (real) sympletic semigroup, since this semigroup has found applications in a variety of settings (see e.g., [4] and [20]).

\section{Subtangent Vectors and Sets.}

We recall certain basic notions concerning subtangent vectors from [8]. Let $S$ be a subset of a finite-dimensional real vector space $V$. A function $\alpha: D \rightarrow \bar{S}$ with $D \subseteq[0, \infty)=\mathbb{R}^{+}, \alpha(D \cap] 0, \infty[) \subseteq S$, is said to be right-differentiable at the origin or, more briefly, 0-right-differentiable provided the following conditions are satisfied:

- 0 is a cluster point of the set of positive numbers in $D$ and $0 \in D$.

- The following limit exists in $V: \dot{\alpha}_{+}(0):=\lim \{(1 / h)(\alpha(h)-\alpha(0))$ : $h \searrow 0, h \in D\}$. 
The limit $\dot{\alpha}_{+}(0)$ is called the right-derivative of $\alpha$ at 0 .

A vector $v \in V$ is called a subtangent vector of $S$ at $x \in V$ if there exists $\alpha: D \rightarrow \bar{S}$ with $\alpha(0)=x$ and satisfying the previous conditions such that $v=\dot{\alpha}_{+}(0)$. The set of all subtangent vectors of $S$ at $x$ is called the subtangent set of $S$ at $x$, and is denoted $\mathcal{L}_{x}(S)$. Note (using the fact that differentiability at 0 implies continuity at 0 ) that the definition is local in the sense that $\mathcal{L}_{x}(S)=\mathcal{L}_{x}(S \cap U)$ for any open set $U$ containing $x$. The definition easily generalizes to smooth manifolds (for our purposes "smooth" will mean $C^{\infty}$ ) over $V$ by passing to $V$ via a chart. Thus for a subset $S$ of a smooth manifold $M$ and $x \in M$, we can define $\mathcal{L}_{x}(S)$ to be all subtangent vectors $\dot{\alpha}_{+}(0)$, where $\alpha: D \rightarrow M$ is as above with $\alpha(0)=x$ and the derivative $\dot{\alpha}(0)$ is computed in any chart. The chain rule and the fact that the definition is local yield that the limit exists and is well-defined if and only if it exists in any chart.

The following appears as Proposition I.5.3 in [8].

Proposition 2.1. Let $\mathbf{C}$ be a closed convex cone in a finite-dimensional real vector space $V$. Then, for $x \in \mathbf{C}, \mathcal{L}_{x}(\mathbf{C})=\overline{\mathbf{C}-\mathbb{R}^{+} x}=\overline{\mathbf{C}+\mathbb{R} x}$.

Let $M$ be a smooth manifold and let $X: M \rightarrow T M$ be a smooth vector field. There exists a corresponding local flow $\Phi$ defined on an open subset of $\mathbb{R} \times M$ containing $\{0\} \times M$ into $M$. We say that a subset $S$ of $M$ is (fully) invariant under the flow generated by $X$ if $\Phi(t, x) \in S$ whenever $x \in S$, $t \geq 0$, and $\Phi(t, x)$ is defined. The Invariance Theorem for Vector Fields (see, e.g., Theorem I.5.17 of [8]) gives an important connection between invariant sets and subtangent sets.

Theorem 2.2. Let $V$ be a finite-dimensional real vector space, let $M$ be a smooth manifold over $V$, and let $X: M \rightarrow T M$ be a smooth vector field over $M$. Let $S$ be a closed subset of $M$. The following conditions are equivalent:

(1) $S$ is invariant under the flow generated by $X$.

(2) For all $m \in S, X(m) \in \mathcal{L}_{m}(S)$.

\section{Lie Semigroup Theory.}

The Lie theory of semigroups denotes primarily the theory of closed infinitesimally generated subsemigroups of Lie groups. These are the ones for which the data of the Lie algebra are sufficient for the reconstruction of the semigroup. Major treatments of the theory can be found in the monographs [8], [9], and [11]. In this section we give a quick recap of some basic Lie semigroup theory that will be useful in the sequel.

In a Banach space $E$ a (closed) wedge is a non-empty closed subset $\mathbf{W}$ of $E$ satisfying

- $\mathbf{W}+\mathbf{W} \subseteq \mathbf{W}$;

- $\mathbb{R}^{+} . \mathbf{W} \subseteq \mathbf{W}$, where $\mathbb{R}^{+}=[0, \infty)$. 
We often employ the terminology "wedge" instead of the more usual "closed convex cone" when we want to emphasize the (potential) presence of a nontrivial edge $\mathbf{W} \cap-\mathbf{W}$, the largest subspace contained in $\mathbf{W}$.

Definition 3.1. If $\mathfrak{g}$ is a (finite dimensional) real or complex Lie algebra, $\mathbf{W}$ is a closed wedge in $\mathfrak{g}$, and the edge $\mathbf{W} \cap-\mathbf{W}$ is a Lie subalgebra of $\mathfrak{g}$, then $\mathbf{W}$ is called a Lie wedge or a Lie-Loewner wedge if $\exp (\operatorname{ad} X)(\mathbf{W}) \subseteq \mathbf{W}$ for each $X \in \mathbf{W} \cap-\mathbf{W}$, the edge of $\mathbf{W}$.

Definition 3.2. Let $G$ be a Lie group, exp $: \mathfrak{g} \rightarrow G$. Let $S$ be a subsemigroup of $G$ containing the identity $e$. The subtangent set of $S$ is

$$
\begin{array}{ccc}
\mathbf{L}(S):= & \{X \in \mathfrak{g}: \forall t \geq 0, \exp (t X) \in S\} \\
& =\left\{\dot{\alpha}(0): \alpha: D \subseteq \mathbb{R}^{+} \rightarrow G, 0 \in D, \alpha(0)=e, \alpha(D) \subseteq S\right\}=\mathcal{L}_{e}(S) .
\end{array}
$$

(It is not obvious that the two sets on the right are equal, but it is a standard result in the Lie theory of semigroups.)

Theorem 3.3. If $S \subseteq G$ is a closed subsemigroup containing e, then the subtangent set $\mathbf{L}(S)$ is a Lie-Loewner wedge, and ${\overline{\langle\exp \mathbf{L}(S)\rangle_{\mathrm{sgp}}}}_{\text {, the closure }}$ of the subsemigroup generated by the exponential image of the subtangent wedge of $S$, is contained in $S$.

Definition 3.4. A subsemigroup $S$ of a Lie group $G$ is infinitesimally generated if the one-parameter semigroups contained in $S$ generate a dense subsemigroup of $S$. If $S$ is a closed subsemigroup, this is equivalent to saying that

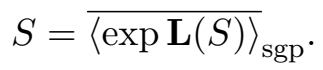

\section{Symmetric Algebras and Ol'shanski Semigroups.}

Let $G$ be a finite-dimensional Lie group with Lie algebra $\mathfrak{g}$ and let $\tau: G \rightarrow G$ be a differentiable involution of $G$, where $\tau$ is assumed distinct from the identity. The pair $(G, \tau)$ is called an involutive group. Then the derivative of $\tau$ at the identity $e, \dot{\tau}: \mathfrak{g} \rightarrow \mathfrak{g}$, is a Lie algebra involution and leads to a decomposition of $\mathfrak{g}$ into the +1 -eigenspace $\mathfrak{h}$ and the -1-eigenspace $\mathfrak{q}, \mathfrak{g}=\mathfrak{h}+\mathfrak{q}$, which satisfies

$$
[\mathfrak{h}, \mathfrak{h}] \subseteq \mathfrak{h},[\mathfrak{h}, \mathfrak{q}] \subseteq \mathfrak{q},[\mathfrak{q}, \mathfrak{q}] \subseteq \mathfrak{h}
$$

(Lie algebras with a given decomposition with these properties are called symmetric algebras.) Let $H$ be a subgroup of $G$ such that $\left(G^{\tau}\right)_{0} \subseteq H \subseteq G^{\tau}$, where $G^{\tau}$ is the (closed) fixed-point group of $\tau$ and $\left(G^{\tau}\right)_{0}$ is its identity component. It follows readily that $H$ is closed and has Lie algebra $\mathfrak{h}$. If there is a closed convex cone $\mathbf{C}$ in $\mathfrak{q}$ which is $\operatorname{Ad}(H)$-invariant and if each $\operatorname{ad}(X), X \in \mathbf{C}$, has real spectrum, then under quite general conditions the set $S:=H \exp (\mathbf{C})=\exp (\mathbf{C}) H$ is a closed infinitesimally generated 
subsemigroup of $G$ with $\mathbf{L}(S)=\mathfrak{h}+\mathbf{C}$. The following is the principal theorem of $[\mathbf{1 5}]$.

Theorem 4.1. Let $(G, \tau)$ be a finite dimensional Lie group, and let $H$ be a closed subgroup of the fixed-point group $G^{\tau}$ containing the identity component of $G^{\tau}$. Let $\mathfrak{g}=\mathfrak{h}+\mathfrak{q}$ be the corresponding symmetric decomposition of the Lie algebra of $G$, and let $\mathfrak{z}$ denote the center of $\mathfrak{g}$. Let $\mathbf{C}$ be a closed convex cone in $\mathfrak{q}$ which is invariant under the adjoint action of $H$, and for which $\operatorname{ad}(X)$ has real spectrum for each $X \in \mathbf{C}$. Then the following are equivalent.

(1) $(X, h) \mapsto(\exp X) h: \mathbf{C} \times H \rightarrow(\exp \mathbf{C}) H$ is a homeomorphism (diffeomorphism) onto a closed subset of $G$.

(2) The mapping $\operatorname{Exp}: \mathfrak{q} \rightarrow G / H$ defined by $\operatorname{Exp}(X)=(\exp X) H$ restricted to $\mathbf{C}$ is a homeomorphism (diffeomorphism) onto a closed subset of $G / H$.

(3) The mapping exp restricted to $\mathbf{C}$ is a homeomorphism (diffeomorphism) onto a closed subset of $G$.

(4) (i) If $Z \in \mathfrak{z} \cap(\mathbf{C}-\mathbf{C})$ satisfies $\exp Z=e$, then $Z=0$.

(ii) For each nonzero $X \in \mathbf{C} \cap \mathfrak{z}$, the closure of $\exp (\mathbb{R} X)$ is not compact. If these conditions hold, then $S:=(\exp \mathbf{C}) H$ is a closed semigroup with subtangent wedge $\mathcal{L}(S)=\mathbf{C}+\mathfrak{h}$. Furthermore the factorization of $s \in S$ is uniquely given by $s=\exp (X) h$, where $X$ is the unique member of $\mathbf{C}$ such that $\exp 2 X=s(\tau(s))^{-1}$ and $h=\exp (-X) s$.

Semigroups satisfying the conditions of Theorem 4.1 originated in a seminal paper of Ol'shanski [19] and they are thus called Ol'shanski semigroups. For a survey of Ol'shanski semigroups, see [16].

Suppose that the hypotheses of Theorem 4.1 are satisfied and that $S=$ (exp C) $H$ is an Ol'shanski semigroup in $G$. Suppose further that the cone $\mathbf{C}$ is pointed (that is, $\mathbf{C} \cap-\mathbf{C}=\{0\}$ ) and generates $\mathfrak{q}$ in the sense that $\mathbf{C}+(-\mathbf{C})=\mathfrak{q}$. There exists a well-defined closed partial order on the coset space $G / H$ given by

$$
a H \leq b H \Leftrightarrow a^{-1} b \in S .
$$

(The fact that $\mathbf{C}$ is pointed yields that the group of units of $S$ is precisely $H$, which in turns gives the antisymmetry of the order.) If $a H \leq b H$ in $G / H$, then the order interval $[a H, b H]$ consists of all $g H$ such that $a H \leq g H \leq b H$. The order is said to be globally hyperbolic if each order interval is compact.

Theorem 4.2. Suppose that the hypotheses of Theorem 4.1 are satsified and that $\mathbf{C}$ is pointed and generates $\mathfrak{q}$. Then the induced order on $G / H$ is globally hyperbolic if and only if for every pair of sequences $\left\{s_{n}\right\}$ in $\exp \mathbf{C}$ and $\left\{t_{n}\right\}$ in $S$ such that the sequence $\left\{s_{n} t_{n}\right\}$ is convergent, some subsequence of $\left\{s_{n}\right\}$ is also convergent. 
Proof. Let the order be globally hyperbolic and let $s_{n} t_{n} \rightarrow g$, where $s_{n} \in$ $\exp \mathbf{C}, t_{n} \in S$ for each $n$. Since $S=(\exp \mathbf{C}) H$ is a closed subsemigroup, $g \in S$. Since $\mathbf{C}$ generates $\mathfrak{q}$, it has dense interior $\mathbf{C}^{\circ}$ in $\mathfrak{q}$. It then follows from Theorem 4.1 and dimensional considerations that that the interior of $S$ in $G$ is $\left(\exp \mathbf{C}^{\circ}\right) H$. Pick $u$ in the interior of $S$. Since $t_{n}^{-1} s_{n}^{-1} g u \rightarrow u$, we conclude that $q_{n}:=t_{n}^{-1} s_{n}^{-1} g u$ is in the interior of $S$ for $n$ large enough. Thus $H=e H \leq s_{n} H \leq s_{n} t_{n} H \leq g u H$ for large $n$. Since the order interval $[e H, g u H]$ is compact, some subsequence of $s_{n} H$ converges to some $w H$ in $G / H$ (we denote the subsequence again by $s_{n}$ ). By Theorem 4.1 there exist $X_{n}, W \in \mathbf{C}$ such that $\operatorname{Exp} X_{n}=s_{n} H$ and $\operatorname{Exp} W=w H$ and $X_{n} \rightarrow W$. Now $\left(\exp X_{n}\right) H=\operatorname{Exp} X_{n}=s_{n} H$ implies that $s_{n}=\left(\exp X_{n}\right) h_{n}$ for some $h_{n} \in H$; by uniqueness of factorization in $S$ we have $s_{n}=\exp X_{n} \rightarrow \exp W$.

To establish the converse, it suffices to consider only intervals of the form $[e H, s H]$ for $s \in S$, since every order interval is a (left) translate of one of this type and the translations preserve the order. By Theorem 4.1 each member of this interval has the form $(\exp X) H$ for some $X \in \mathbf{C}$, and it must be the case that $(\exp X)^{-1} s \in S$. Let $\left\{X_{n}\right\}$ be a sequence in $\mathbf{C}$ with $\left(\exp X_{n}\right) H \in[e H, s H]$ for each $n$. Then the sequence $\exp X_{n}\left(\left(\exp X_{n}\right)^{-1} s\right)$ is the constant sequence converging to $s$; hence by hypothesis the sequence $\exp X_{n}$ has a convergence subsequence, and hence so does $\left(\exp X_{n}\right) H$. Thus the interval is compact.

Remark 4.3. Note that $\operatorname{Exp}: \mathbf{C} \rightarrow \operatorname{Exp}(\mathbf{C})=S / H$ is a bijection for all choices of $H$. We remark that the order on $\operatorname{Exp}(\mathbf{C})$ is also independent of the choice of $H$. Thus any of the ordered homogeneous spaces $S / H$ induces a unique partial order on $\mathbf{C}$ via the inverse of Exp such that Exp : $\mathbf{C} \rightarrow \operatorname{Exp}(\mathbf{C})=S / H$ is an order isomorphism.

Proof. Let $H_{0}$ denote the identity component of $H$; note that $H_{0}=\left(G^{\tau}\right)_{0}$. Clearly $a H_{0} \leq b H_{0}$ implies that $a H \leq b H$ since $(\exp \mathbf{C}) H_{0} \subseteq(\exp \mathbf{C}) H$. Conversely suppose $(\operatorname{Exp} X) H \leq(\operatorname{Exp} Y) H$. Then $(\exp X)^{-1}(\exp Y)=$ $(\exp W) h \in(\exp \mathbf{C}) H$, or $\exp Y=(\exp X)(\exp W) h$. Since $(\exp \mathbf{C}) H_{0}$ is an Ol'shanski semigroup, we can rewrite $(\exp X)(\exp W)=(\exp U) g$ for some $g \in H_{0}$. Then $\exp Y=(\exp U) g h$ and by uniqueness of factorization in $(\exp \mathbf{C}) H, g h=e$, and thus $h \in H_{0}$.

Let $(\mathfrak{g}, \tau)$ be a symmetric algebra, $\mathfrak{g}=\mathfrak{h}+\mathfrak{q}$. An element $X \in \mathfrak{g}$ is called hyperbolic if the spectrum of $\operatorname{ad} X$ is real and $\operatorname{ad} X$ is semisimple (i.e., diagonalizable) as a linear operator. A cone (always assumed closed and convex) $\mathbf{C}$ in $\mathfrak{q}$ has dense interior in the vector space $\mathbf{C}-\mathbf{C}$, and if $\operatorname{ad} X$ is hyperbolic for each $X$ in the interior of $\mathbf{C}$, then the cone is said to be hyperbolic. Note by continuity of spectrum that $\operatorname{ad} X$ has real spectrum for all $X$ in a hyperbolic cone. The symmetric algebra $\mathfrak{g}$ is said to be effective if $X \in \mathfrak{h}$ and $[X, Y]=0$ for all $Y \in \mathfrak{q}$ implies $X=0$; this is equivalent to saying that $\mathfrak{q}$ is an effective $\mathfrak{h}$-module. 
Theorem 4.4. Let $(G, \tau)$ be an involutive Lie group and let $H$ be a closed subgroup with Lie algebra $\mathfrak{h}$. Suppose that $\mathbf{C} \subseteq \mathfrak{q}$ is a pointed generating $\mathrm{Ad} H$-invariant hyperbolic cone. Then the order on $G / H$ is globally hyperbolic.

Proof. By translation it suffices to prove that order intervals in $\operatorname{Exp}(\mathbf{C})$ are compact. By the previous remark the order there is independent of the choice of $H$. Thus we choose $H$ to be the (closed) connected Lie subgroup with Lie algebra $\mathfrak{h}$. Since this is contained in any choice of $H$, the cone $\mathbf{C}$ will remain $\mathrm{Ad} H$-invariant. But in the connected case, this is the same as requiring that $\mathbf{C}$ be invariant under $\langle\exp (\operatorname{adh})\rangle$, the subgroup of inner automorphisms of $\mathfrak{g}$ generated by $\exp (\operatorname{ad} \mathfrak{h})$.

We first assume that the symmetric algebra $\mathfrak{g}$ of $G$ is effective. We consider the complexification $\mathfrak{g}_{\mathbb{C}}=\mathfrak{g} \oplus i \mathfrak{g}$; it is a symmetric Lie algebra with respect to the structure $\hat{\mathfrak{h}}=\mathfrak{h}+i \mathfrak{q}$ and $\hat{\mathfrak{q}}=\mathfrak{q}+i \mathfrak{h}$. We apply the Extension Theorem, Theorem X.7 of [13] (together with Theorem X.3, Propositions V.9 and VII.12, Theorem VIII.1, and Corollary IX.10 to verify that the hypotheses of the theorem are satisfied). The Extension Theorem states that there exists a pointed generating hyperbolic cone $\hat{\mathbf{C}}$ in $\hat{\mathfrak{q}}$ which is invariant under the inner automorphism group $\langle\exp (\operatorname{ad} \hat{\mathfrak{h}})\rangle$ such that $\hat{\mathbf{C}} \cap \mathfrak{q}=\mathbf{C}$. The Extension Theorem is all that one needs in order to use the proof in Section 5.3 of [10] that the order on $G / H$ is globally hyperbolic.

For the general case in which the symmetric algebra is not effective, consider the subgroup $L$ of $H$ which is the kernel of the representation $\left.H \rightarrow \operatorname{Ad} H\right|_{\mathfrak{q}}$; then $L$ is normal in $H$. Since $L$ is also the subgroup of $H$ which centralizes $\exp \mathfrak{q}$ and since the union of $\exp \mathfrak{q}$ and $H$ generate $G$, we conclude that $L$ is normal in $G$. Then $G / H \approx(G / L) /(H / L)$ and the restriction of the identification identifies the image $\operatorname{Exp}(\mathbf{C})$ in each and also is an order isomorphism (as can be easily verified). Since $L \subseteq H$, there is a unique involution on $G / L$ such that the natural homomorphism is a homomorphism of involutive groups. The preceding case of an effective action can now be applied to $G / L$ to conclude that the order on $\operatorname{Exp}(\mathbf{C})$ in $(G / L) /(H / L)$ and hence in $G / H$ has compact intervals, and thus that the order in $G / H$ is globally hyperbolic.

Remark 4.5. We remark that the proof actually yields more than that the intervals are compact. It shows that when the partial order on $S / H$ is pulled back to $\mathbf{C}$ via $\operatorname{Exp}^{-1}$, then the order is contained in the standard order arising from $\mathbf{C}$ defined by $x \leq y$ if and only if $y-x \in \mathbf{C}$.

\section{Cayley-type Symmetric Algebras.}

Let $(G, \tau)$ be an involutive Lie group, i.e., a connected Lie group equipped with a differentiable involution $\tau$ which is not the identity. Let $\dot{\tau}: \mathfrak{g} \rightarrow \mathfrak{g}$ 
be the derivative at the identity, and let $\mathfrak{h}$ resp. $\mathfrak{q}$ be the +1 resp. -1 eigenspace. The symmetric algebra $\mathfrak{g}$ is called a symmetric algebra of Cayley type if there exist abelian subalgebras $\mathfrak{q}_{-}$and $\mathfrak{q}_{+}$of $\mathfrak{g}$ contained in $\mathfrak{q}$ such that $\mathfrak{q}=\mathfrak{q}_{-} \oplus \mathfrak{q}_{+}$. Note that the triple $\left(\mathfrak{q}_{-}, \mathfrak{h}, \mathfrak{q}_{+}\right)$is a $(-1,0,1)$-graded Lie algebra.

Let $H$ be a closed subgroup of $G$ with Lie algebra $\mathfrak{h}$. We define a smooth mapping

$$
\phi: \mathfrak{q}_{-} \times H \times \mathfrak{q}_{+} \rightarrow G, \quad \phi(X, h, Y)=(\exp X) h(\exp Y) .
$$

Recall (see, for example, Chapter 2 of [7]) that the derivative of the exponential function $\exp : \mathfrak{g} \rightarrow G$ at $X \in \mathfrak{g}$ is given by $d_{X} \exp =d_{e} \lambda_{\exp X} \circ$ $f(\operatorname{ad} X)$, where $f(\operatorname{ad} X): \mathfrak{g} \rightarrow \mathfrak{g}$ is given by plugging the operator ad $X$ into the series

$$
f(T)=\frac{1-e^{-T}}{T}=\sum_{n=0}^{\infty} \frac{(-1)^{n}}{(n+1) !} T^{n} .
$$

Lemma 5.1. Suppose that $X \in \mathfrak{q}_{-}, h \in H, Y \in \mathfrak{q}_{+}$. Then $\phi$ is regular at $(X, h, Y)$.

Proof. We first suppose that $h=e$, the identity. We denote the linear mapping $d \phi$ at $(X, e, Y)$ by $d_{(X, e, Y)} \phi$. Then for $u_{-} \in \mathfrak{q}_{-}, u \in \mathfrak{h}, u_{+} \in \mathfrak{q}_{+}$, $\left(u_{-}, u, u_{+}\right) \in T_{(X, e, Y)}\left(\mathfrak{q}_{-} \times H \times \mathfrak{q}_{+}\right)$, and the following equation holds:

$$
\begin{aligned}
& d \phi_{(X, e, Y)}\left(u_{-}, u, u_{+}\right) \\
& =d \phi_{(X, e, Y)}\left(u_{-}\right)+d_{(X, e, Y)}(u)+d_{(X, e, Y)}\left(u_{+}\right) \\
& =\left.d_{\exp X} \rho_{\exp Y} \circ d_{X} \exp \right|_{\mathfrak{q}_{-}}\left(u_{-}\right)+d_{\exp Y} \lambda_{\exp X} \circ d_{e} \rho_{\exp Y}(u) \\
& \quad+\left.d_{\exp Y} \lambda_{\exp X} \circ d_{Y} \exp \right|_{\mathfrak{q}_{+}}\left(u_{+}\right) \\
& =d_{\exp Y} \lambda_{\exp X} \circ d_{e} \rho_{\exp Y} \circ f(\operatorname{ad} X)\left(u_{-}\right)+d_{\exp Y} \lambda_{\exp X} \circ d_{e} \rho_{\exp Y}(u) \\
& \quad+d_{\exp Y} \lambda_{\exp X} \circ d_{e} \lambda_{\exp Y} \circ f(\operatorname{ad} Y)\left(u_{+}\right) \\
& =d_{\exp Y} \lambda_{\exp X}\left(d_{e} \rho_{\exp Y} \circ f(\operatorname{ad} X)\left(u_{-}\right)\right. \\
& \left.\quad+d_{e} \rho_{\exp Y}(u)+d_{e} \lambda_{\exp Y} \circ f(\operatorname{ad} Y)\left(u_{+}\right)\right) .
\end{aligned}
$$

If $d \phi_{(X, e, Y)}\left(u_{-}, u, u_{+}\right)=0$, then since $\lambda_{\exp X}$ is a diffeomorphism,

$$
d_{e} \rho_{\exp Y}\left(u+f(\operatorname{ad} X) u_{-}\right)=-d_{e} \lambda_{\exp Y}\left(f(\operatorname{ad} Y)\left(u_{+}\right)\right)
$$

or

$$
u+f(\operatorname{ad} X)\left(u_{-}\right)=-\operatorname{Ad}(\exp Y)\left(f(\operatorname{ad} Y)\left(u_{+}\right)\right)=-e^{\operatorname{ad} Y} \circ f(\operatorname{ad} Y)\left(u_{+}\right) .
$$

Since $\left.\operatorname{ad} X\right|_{\mathfrak{q}_{-}}=0$ and $\left.\operatorname{ad} Y\right|_{\mathfrak{q}_{+}}=0$, we obtain $u+u_{-}=-u_{+}$. Since each lives in a distinct direct summand of $\mathfrak{g}$, we conclude that $u=u_{+}=u_{-}=0$. Thus $d \phi_{(X, e, y)}$ is injective, hence (by dimensional considerations) surjective, and thus $d \phi$ is regular at $(X, e, Y)$. 
For the general case, we need to show that $\phi$ is regular at $(X, h, Y)$ for arbitrary $h \in H$. Fix $h \in H$ and consider the composition

$$
\begin{aligned}
(X, g, Y) \rightarrow & \left(X, g h^{-1}, \operatorname{Ad} h(Y)\right) \stackrel{\phi}{\rightarrow} \phi\left(X, g h^{-1}, \operatorname{Ad} h(Y)\right) \\
= & (\exp X) g h^{-1} \exp ((\operatorname{Ad} h) Y) \\
& \stackrel{\rho_{h}}{\rightarrow}(\exp X) g h^{-1} \exp ((\operatorname{Ad} h) Y) h \\
= & (\exp X) g(\exp Y)=\phi(X, g, Y) .
\end{aligned}
$$

The first mapping is a diffeomorphism since right translation by $h^{-1}$ is a diffeomorphism and $\mathrm{Ad} h$ is linear and the third mapping $\rho_{h}$ is also a diffeomorphism. Beginning at $(X, h, Y)$ (that is, with $g=h)$ we obtain from the first part of the proof that $\phi$ is regular at the point $\left(X, e=h h^{-1}, \operatorname{Ad} h(Y)\right)$. It follows that the composition $\phi$ is regular at $(X, h, Y)$.

The authors are indebted to G. Ólafsson for pointing out that the following theorem should be true.

Theorem 5.2. Let $(G, \tau)$ be an involutive Lie group such that the Lie algebra $\mathfrak{g}=\mathfrak{q}_{-}+\mathfrak{h}+\mathfrak{q}_{+}$is a symmetric algebra of Cayley type. Let $H$ be a closed subgroup of $G^{\tau}$ with Lie algebra $\mathfrak{h}$. Suppose further that $0 \neq X \in \mathfrak{z} \cap \mathfrak{q}$ implies $\exp (X) \neq e$. Then the mapping $\phi$ of equation $(\dagger)$ is a diffeomorphism onto an open subset of $G$.

Proof. It follows from the Inverse Mapping Theorem and the preceding lemma that $\phi$ is a local diffeomorphism onto an open subset of $G$. Hence we need only to show that $\phi$ is injective. Suppose that $\left(\exp X_{1}\right) h_{1}\left(\exp Y_{1}\right)=$ $\left(\exp X_{2}\right) h_{2}\left(\exp Y_{2}\right)$. Then using that fact that $\mathfrak{q}_{-}$and $\mathfrak{q}_{+}$are abelian, we obtain

$$
g_{1}:=\exp \left(X_{1}-X_{2}\right) h_{1}=h_{2} \exp \left(Y_{2}-Y_{1}\right)=\exp \left(\left(\operatorname{Ad} h_{2}\right)\left(Y_{2}-Y_{1}\right)\right) h_{2}:=g_{2} \text {. }
$$

Setting $g^{\sharp}:=(\tau(g))^{-1}$, we have

$$
g_{1} g_{1}^{\sharp}=\exp \left(X_{1}-X_{2}\right) h_{1}\left(h_{1}\right)^{-1} \exp \left(X_{1}-X_{2}\right)=\exp \left(2 X_{1}-2 X_{2}\right),
$$

and similarly $g_{2} g_{2}^{\sharp}=\exp \left(\left(\operatorname{Ad} h_{2}\right)\left(2 Y_{2}-2 Y_{1}\right)\right)$.

Now $X:=2 X_{1}-2 X_{2} \in \mathfrak{q}_{-}$and $Y:=\operatorname{Ad}_{2}\left(2 Y_{1}-2 Y_{2}\right) \in \mathfrak{q}_{+}$, from the preceding paragraph $\exp X=\exp Y$, and thus

$$
e^{\operatorname{ad} X}=\operatorname{Ad}(\exp X)=\operatorname{Ad}(\exp Y)=e^{\operatorname{ad} Y} .
$$

Suppose that $X$ is not in $\mathfrak{z}$. Then $[X, V] \neq 0$ for some $V$ in $\mathfrak{h}$ or $\mathfrak{q}_{+}$. If $V \in \mathfrak{h}$, then $e^{\operatorname{ad} X}(V)$ has $\mathfrak{q}_{-}$-component $[X, V]$, but $e^{\operatorname{ad} Y}(V)$ is contained in $\mathfrak{h}+\mathfrak{q}_{+}$, a contradiction. If $V \in \mathfrak{q}_{+}$, the $e^{\operatorname{ad} X}(V)$ had $\mathfrak{h}$-component $[X, V]$, but $e^{\operatorname{ad} Y}(V)=V \in \mathfrak{q}_{+}$, again a contradiction. Thus $X \in \mathfrak{z}$, and similarly $Y \in \mathfrak{z}$. But then $Y-X \in \mathfrak{z} \cap \mathfrak{q}$ and $\exp (Y-X)=e$, a contradiction to the hypothesis of the theorem, unless $X=Y=0$. We conclude that $X_{1}=X_{2}$ and $Y_{1}=Y_{2}$, and it then follows immediately that $h_{1}=h_{2}$. 
Theorem 5.3. Let $(G, \tau)$ be an involutive Lie group such that the Lie algebra $\mathfrak{g}=\mathfrak{q}_{-}+\mathfrak{h}+\mathfrak{q}_{+}$is a symmetric algebra of Cayley type. Let $H$ be a closed subgroup of $G^{\tau}$ with Lie algebra $\mathfrak{h}$. Suppose that $\mathbf{C}^{-}$is a cone in $\mathfrak{q}_{-}$and $\mathbf{C}^{+}$ is a cone in $\mathfrak{q}_{+}$such that $\mathbf{C}:=\mathbf{C}^{+}+\mathbf{C}^{-}$is a hyperbolic $\operatorname{Ad}(H)$-invariant cone. Set $S:=\left(\exp \mathbf{C}^{-}\right) H\left(\exp \mathbf{C}^{+}\right)$. If any of the conditions (1)-(4) of Theorem 4.1 is satisfied, then the mapping

$$
\phi: \mathbf{C}^{-} \times H \times \mathbf{C}^{+} \rightarrow S, \quad \phi(X, h, Y)=(\exp X) h(\exp Y)
$$

is a diffeomorphism. If further the set $S$ is closed, then $S$ is a semigroup and equal to the Ol'shanski semigroup $(\exp \mathbf{C}) H$. The set $S$ is closed and the conclusions follow in the case that $\mathbf{C}$ is pointed.

Proof. For the proof of the theorem, it suffices to redefine (if necessary) $\mathfrak{q}_{-}$to be $\mathbf{C}^{-}-\mathbf{C}^{-}$and $\mathfrak{q}_{+}$to be $\mathbf{C}^{+}-\mathbf{C}^{+}$. The invariance of $\mathbf{C}$ and the original $\mathfrak{q}_{-}$and $\mathfrak{q}_{+}$under $\operatorname{Ad}(H)$ implies that under this redefinition we again obtain a symmetric algebra of Cayley type. From condition (4) of Theorem 4.1 and the preceding Theorem 5.2 we conclude that the mapping $\phi$ is a diffeomorphism from all of $\mathfrak{q}_{-} \times H \times \mathfrak{q}_{+}$onto an open subset of $G$, and hence its restriction from $\mathbf{C}^{-} \times H \times \mathbf{C}^{+}$onto $S$ is a diffeomorphism.

Assume now that $S$ is closed. We deduce from Theorem 4.1 that $T:=$ $H(\exp \mathbf{C})=(\exp \mathbf{C}) H, S^{-}:=H\left(\exp \mathbf{C}^{-}\right)=\left(\exp \mathbf{C}^{-}\right) H$, and $S^{+}=$ $H\left(\exp \mathbf{C}^{+}\right)=\left(\exp \mathbf{C}^{+}\right) H$ are all Ol'shanski subsemigroups of $G\left(\mathbf{C}^{-}=\right.$ $\mathbf{C} \cap \mathfrak{q}_{-}$is $\operatorname{Ad}(H)$-invariant since $\mathbf{C}$ and $\mathfrak{q}_{-}$are and similarly for $\left.\mathbf{C}^{+}\right)$. Note that $S^{-} S=S^{-} S^{-}\left(\exp \mathbf{C}^{+}\right)=S^{-}\left(\exp \mathbf{C}^{+}\right)=S$; similarly $S S^{+}=S$. For $h \in H, h S=h S^{-} S=h H\left(\exp \mathbf{C}^{-} S\right)=S^{-} S=S$, and similarly $S h=S$. Thus $H s H \subseteq S$ for all $s \in S$.

Let $X \in \mathbf{C}^{-}$. We claim that $S \exp (t X) \subseteq S$ for all $t \geq 0$. There is a unique left invariant vector field $\tilde{X}(g):=d_{e} \lambda_{g}(X)$ on $G$ corresponding to $X \in \mathbf{C}^{-} \subseteq \mathfrak{g}$. Its flow is given by $(t, g) \mapsto g \exp (t X)$. To prove the claim, it suffices to show that $S$ is invariant under this flow. For this purpose we use the Invariance Theorem 2.2.

Let $q:=\left(\exp X^{\prime}\right) h(\exp Y) \in S$. Then

$$
\begin{aligned}
q=\left(\exp X^{\prime}\right) h(\exp Y) & =h h^{-1}\left(\exp X^{\prime}\right) h(\exp Y) \\
& =h \exp \left(\left(\operatorname{Ad} h^{-1}\right) X^{\prime}\right)(\exp Y) .
\end{aligned}
$$

Note that $s:=\exp \left(\left(\operatorname{Ad} h^{-1}\right) X^{\prime}\right)(\exp Y) \in\left(\exp \mathbf{C}^{-}\right)\left(\exp \mathbf{C}^{+}\right)$since $\mathbf{C}^{-}$is $\operatorname{Ad}(H)$-invariant. Since $\lambda_{h}$ is a diffeomorphism,

$$
\mathcal{L}_{q}(S)=\mathcal{L}_{h s}(S)=d_{s} \lambda_{h}\left(\mathcal{L}_{s}\left(h^{-1} S\right)\right)=d_{s} \lambda_{h}\left(\mathcal{L}_{s}(S)\right) .
$$

Since $\tilde{X}$ is left-invariant, it thus suffices to check that $\tilde{X}(s) \in \mathcal{L}_{s}(S)$ for all $s \in\left(\exp \mathbf{C}^{-}\right)\left(\exp \mathbf{C}^{+}\right)$.

Thus let $s=\left(\exp X^{\prime}\right)(\exp Y) \in\left(\exp \mathbf{C}^{-}\right)\left(\exp \mathbf{C}^{+}\right)$. By Lemma $5.1 \phi$ is regular at $\left(X^{\prime}, e, Y\right)$. By the Inverse Function Theorem there exists an open 
set $U$ containing $s$ and a diffeomorphism $\psi: U \rightarrow \psi(U) \subseteq \mathfrak{q}_{-} \times H \times \mathfrak{q}_{-}$with $\psi(s)=\left(\exp X^{\prime}, e, \exp Y\right)$ such that $\phi \circ \psi$ is the identity on $U$. To show that $\tilde{X}(s) \in \mathcal{L}_{s}(S)$, it suffices to show that

$$
\begin{aligned}
d_{s} \psi(\tilde{X}(s)) \in \mathcal{L}_{\psi(s)}(\psi(S \cap U)) & =\mathcal{L}_{\left(X^{\prime}, e, Y\right)}\left(\left(\mathbf{C}^{-} \times H \times \mathbf{C}^{+}\right) \cap \psi(U)\right) \\
& =\mathcal{L}_{X^{\prime}}\left(\mathbf{C}^{-}\right) \times \mathfrak{h} \times \mathcal{L}_{Y}\left(\mathbf{C}^{+}\right) .
\end{aligned}
$$

Thus we must show $u \in \mathcal{L}_{X^{\prime}}\left(\mathbf{C}^{-}\right)$and $w \in \mathcal{L}_{Y}\left(\mathbf{C}^{+}\right)$, where $d_{s} \psi(\tilde{X}(s))=$ $(u, v, w)$, which transforms to $\left(d_{s} \psi\right)^{-1}(u, v, w)=d_{\left(X^{\prime}, e, Y\right)} \phi(u, v, w)=\tilde{X}(s)$. Applying equation ( $\ddagger)$ of Lemma 5.1, we conclude that $d_{\left(X^{\prime}, e, Y\right)} \phi(u, v, w)$ is equal to

$d_{\exp Y} \lambda_{\exp X^{\prime}}\left(d_{e} \rho_{\exp Y} \circ f\left(\operatorname{ad} X^{\prime}\right)(u)+d_{e} \rho_{\exp Y}(v)+d_{e} \lambda_{\exp Y} \circ f(\operatorname{ad} Y)(w)\right)$.

Since $\tilde{X}(s)=d_{\exp Y} \lambda_{\exp X^{\prime}} \circ d_{e} \lambda_{\exp Y}(X)$, we conclude that

$$
d_{e} \lambda_{\exp Y}(X)=d_{e} \rho_{\exp Y} \circ f\left(\operatorname{ad} X^{\prime}\right)(u)+d_{e} \rho_{\exp Y}(v)+d_{e} \lambda_{\exp Y} \circ f(\operatorname{ad} Y)(w) .
$$

Since $\left(d_{e} \rho_{\exp Y}\right)^{-1} \circ d_{e} \lambda_{\exp Y}=\operatorname{Ad}(\exp Y)$, the preceding transforms to

$$
\operatorname{Ad}(\exp Y)(X)=f\left(\operatorname{ad} X^{\prime}\right)(u)+v+\operatorname{Ad}(\exp Y) \circ f(\operatorname{ad} Y)(w) .
$$

The left-hand side is $\operatorname{Ad}(\exp Y)(X)=e^{\operatorname{ad} Y}(X)=X+[Y, X]+$ $(1 / 2)[Y,[Y, X]]$, while $\left[X^{\prime}, u\right]=0=[Y, w]$ implies that the right-hand side is $u+v+w$. We conclude that $u=X \in \mathbf{C}^{-} \subseteq \mathcal{L}_{X^{\prime}}\left(\mathbf{C}^{-}\right)$and $w=(1 / 2)[Y,[Y, X]]=(1 / 2) \operatorname{ad}([X, Y])(Y)$. Since $[X, Y] \in \mathfrak{h}$, we have $\exp (\operatorname{tad}[X, Y]) Y \in \mathbf{C}^{+}$for all $t \in \mathbb{R}$, and differentiating the curve at $t=$ 0 yields $\operatorname{ad}([X, Y])(Y) \in \mathcal{L}_{Y}\left(\mathbf{C}^{+}\right)$and thus $w=(1 / 2) \operatorname{ad}([X, Y])(Y) \in$ $\mathcal{L}_{Y}\left(\mathbf{C}^{+}\right)$. We conclude from the Invariance Theorem 2.2 that $S \exp \left(\mathbb{R}^{+} X\right) \subseteq$ $S$.

Let $s=(\exp X) h(\exp Y) \in S$. Then

$$
S s=(S(\exp X)) h(\exp Y) \subseteq S S^{+} \subset S ;
$$

thus $S S \subseteq S$, i.e., $S$ is a semigroup.

Clearly $\exp \mathbf{C}^{-}, H$ and $\exp \mathbf{C}^{+}$are subsets of $S$, so $\mathbf{C}^{-}, \mathbf{C}^{+} \subseteq \mathcal{L}(S)$, and hence by Theorem 3.3, $\mathbf{C}=\mathbf{C}^{-}+\mathbf{C}^{+} \subseteq \mathcal{L}(S)$. Again by Theorem 3.3 $\exp \mathbf{C} \subseteq S$, and thus $(\exp \mathbf{C}) H \subseteq S S \subseteq S$. The reverse containment is clear since $(\exp \mathbf{C}) H$ is a semigroup.

Suppose now that additionally $\mathbf{C}$ is pointed. We are assuming (without loss of generality) that (C) generates $\mathfrak{q}$. Then by Theorem 4.4, $\mathbf{C}$ determines a globally hyperbolic order on $G / H$. Let $\left(\exp X_{n}\right) h_{n}\left(\exp Y_{n}\right)$ be a sequence in $S \subseteq(\exp \mathbf{C}) H$ converging to $g$. By Theorem 4.2 some subsequence of $\left\{\exp X_{n}\right\}$ must converge, and then by Theorem $4.1 \exp X_{n} \rightarrow \exp X$ for some $X \in \mathbf{C}^{-}$. Passing to subsequences we conclude that $h_{n} \exp \left(Y_{n}\right)$ converges, and then from Theorem 4.1, $h_{n} \rightarrow h$ and $Y_{n} \rightarrow Y$ for some $h \in H$ and 
some $Y \in \mathbf{C}^{+}$. It follows that $S$ is closed, so the earlier part of the proof applies.

\section{Symmetric Cones, Jordan Algebras, and Tube Domains.}

Given any symmetric cone there is a natural way to associate with it a semigroup of "compressions"; these semigroups form a fundamental class of examples of the theory that has been developed in the previous sections. Symmetric cones can be studied directly as open convex cones in $\mathbb{R}^{n}$ which are self-dual and homogeneous. However, we prefer the (equivalent) approach through the theory of Euclidean Jordan algebras. We first recall basic features of this theory that are needed for our purposes (see, for example, $[6])$.

A commutative algebra $V$ over field $\mathbb{R}$ or $\mathbb{C}$ with product $x y$ is said to be a Jordan algebra if for all elements $x, y$ in $V$,

$$
x\left(x^{2} y\right)=x^{2}(x y) .
$$

A finite dimensional real Jordan algebra $V$ is called an euclidean Jordan algebra if $V$ is a real Hilbert space with an inner product $\langle x \mid y\rangle$ such that for all $x, y, z \in V$

$$
\langle x y \mid z\rangle=\langle y \mid x z\rangle \text {. }
$$

Let $V$ be a euclidean Jordan algebra with identity $e$, and let $Q=\left\{x^{2} \mid x \in\right.$ $V\}$ denote the set of squares. Then the set $Q$ is a self-dual cone and the interior $\Omega$ of $Q$ is a symmetric cone. That is, $\Omega$ is a self-dual cone and the group

$$
G(\Omega)=\{g \in G L(V) \mid g \Omega=\Omega\}
$$

acts on it transitively. Furthermore, the symmetric cone $\Omega$ has the following characterizations.

Theorem 6.1. The symmetric cone $\Omega$ can be alternately characterized as the connected component of e in the set of all invertible elements or as $\exp V$.

We consider the complex Jordan algebra $V_{\mathbb{C}}=V+i V$, the complexification of $V$. The corresponding tube domain $T_{\Omega}:=V+i \Omega$ is a symmetric tube domain which is biholomorphically isomorphic to a bounded symmetric domain via a Cayley transform. Let $G\left(T_{\Omega}\right)$ be the Lie group consisting of all biholomorphic automorphisms of the tube domain $T_{\Omega}$. The group $G\left(T_{\Omega}\right)$ can be described in the following way: an element $g \in G(\Omega)$ acts on the tube domain $T_{\Omega}$ by

$$
z=x+i y \mapsto g(z)=g(x)+i g(y) .
$$

For $x$ in $V$, the translation by $x$

$$
t_{x}: z \longrightarrow z+x
$$


is a holomorphic automorphism of $T_{\Omega}$ and the group of all real translations is an abelian group $N^{+}$isomorphic to the vector space $V$. The map

$$
j: z \mapsto-z^{-1}
$$

is in $G\left(T_{\Omega}\right)$. We set

$$
\tilde{t}_{x}=j \circ t_{x} \circ j
$$

and

$$
N^{-}=j N^{+} j .
$$

Then $G\left(T_{\Omega}\right)$ is generated by $N^{+}, G(\Omega)$ and $j$.

For $x \in V$ or $x \in V_{\mathbb{C}}$ let $L(x)$ denote the (linear) left translation map defined by $L(x) y=x y$, and set $P(x)=2 L(x)^{2}-L\left(x^{2}\right)$. The Lie algebra of $G\left(T_{\Omega}\right)$ can be described in the following way: Let $g_{t}$ be a one-parameter subgroup of $G\left(T_{\Omega}\right)$; then

$$
\tilde{X} f(z)=\left.\frac{d}{d t} f\left(g_{t}(z)\right)\right|_{t=0}, \quad f \in \mathcal{C}^{1}\left(T_{\Omega}\right),
$$

defines a vector field on $T_{\Omega}$, and the set of vector fields obtained in this way is a real Lie subalgebra with respect to the usual Lie bracket of vector fields. We can write

$$
\tilde{X} f(z)=D f(z)(X(z))
$$

where, for each $z, X(z)$ is a vector in $V_{\mathbb{C}}=V+i V$. Then the Lie algebra of $G\left(T_{\Omega}\right)$ is the set of vector fields of the form

$$
X(z)=u+T z+P(z) v,
$$

where $u, v \in V$ and $T \in \mathfrak{g}(\Omega)$, where $\mathfrak{g}(\Omega)$ is the Lie algebra of $G(\Omega)$ (Theorem X.5.10, [6]). A vector field $X$ in $\mathfrak{g}\left(T_{\Omega}\right), X=u+T z+P(z) v$, can be identified with $(u, T, v) \in V \times \mathfrak{g}(\Omega) \times V$. Let

$$
\begin{aligned}
\mathfrak{g}_{-1}\left(T_{\Omega}\right) & =\{(u, 0,0) \mid u \in V\} \cong V, \\
\mathfrak{g}_{0}\left(T_{\Omega}\right) & =\{(0, T, 0) \mid T \in \mathfrak{g}(\Omega)\} \cong \mathfrak{g}(\Omega), \\
\mathfrak{g}_{1}\left(T_{\Omega}\right) & =\{(0,0, v) \mid v \in V\} \cong V .
\end{aligned}
$$

If $X=(u, T, v), X^{\prime}=\left(u^{\prime}, T^{\prime}, v^{\prime}\right)$, and $u \square v=L(u v)+[L(u), L(v)]$, then

$$
\left[X, X^{\prime}\right]=\left(T u^{\prime}-T^{\prime} u, 2 u^{\prime} \square v+\left[T, T^{\prime}\right]-2 u \square v^{\prime}, T^{t} v^{\prime}-T^{\prime t} v\right) .
$$

Let $\theta=\operatorname{Ad}(j)$; then $\theta(u, T, v)=\left(v,-T^{t}, u\right)$ is a Cartan involution. The Cartan decomposition corresponding to $\theta$ is $\mathfrak{k} \oplus \mathfrak{p}$, where

$$
\begin{aligned}
\mathfrak{k} & =\left\{(u, T, u) \mid T^{t}=-T\right\}, \\
\mathfrak{p} & =\left\{(u, T,-u) \mid T^{t}=T\right\} .
\end{aligned}
$$

Define $\eta: \mathfrak{g} \rightarrow \mathfrak{g}$ by $\eta(u, T, v)=(-u, T,-v)$. Then $\eta$ gives rise to a Cayley-type symmetric algebra structure on $\mathfrak{g}$ with

$$
\mathfrak{g}_{0}=\left\{X \in \mathfrak{g}\left(T_{\Omega}\right) \mid \eta X=X\right\},
$$


and

$$
\mathfrak{g}_{-1} \oplus \mathfrak{g}_{1}=\left\{X \in \mathfrak{g}\left(T_{\Omega}\right) \mid \eta X=-X\right\}
$$

Set

$$
\begin{aligned}
\mathfrak{h} & =\mathfrak{g}_{0}, \\
\mathfrak{q} & =\mathfrak{g}_{-1} \oplus \mathfrak{g}_{1}, \\
\mathfrak{n}^{+} & =\mathfrak{g}_{-1}, \\
\mathfrak{n}^{-} & =\mathfrak{g}_{1} .
\end{aligned}
$$

Then

$$
\mathfrak{g}\left(T_{\Omega}\right)=\mathfrak{k} \oplus \mathfrak{p}=\mathfrak{h} \oplus \mathfrak{q}=\mathfrak{n}^{+} \oplus \mathfrak{h} \oplus \mathfrak{n}^{-} .
$$

This is a semisimple hermitian Lie algebra.

Define the real conjugation $\sigma$ on $T_{\Omega}$ by $\sigma(x+i y)=-x+i y$. We can then define an involution $\tau$ on $G\left(T_{\Omega}\right)$ by $\tau(g):=\sigma g \sigma$. One verifies easily that $\tau$ is the identity on the subgroup $G(\Omega)$. We note that $N^{ \pm}$is the Lie subgroup of $G\left(T_{\Omega}\right)$ with Lie algebra $\mathfrak{n}^{ \pm}$. Again one verifies directly that $\tau$ restricted to $N^{+}$is inversion. It then follows easily using the fact that $\sigma$ commutes with $j$ that $\tau$ restricted to $N$ is also inversion. From these facts one readily deduces that the derivative of $\tau$ on the Lie algebra $\mathfrak{g}\left(T_{\Omega}\right)$ is equal to $\eta$. Hence

$$
\mathfrak{g}\left(T_{\Omega}\right)=\mathfrak{n}^{-} \oplus \mathfrak{h} \oplus \mathfrak{n}^{+}
$$

is a Cayley algebra for the involutive group $\left(G\left(T_{\Omega}\right), \tau\right)$.

Since the algebra is semisimple, then by Thereom 5.2 the mapping

$$
(X, h, Y) \in \mathfrak{n}^{+} \times G(\Omega) \times \mathfrak{n}^{-} \longrightarrow(\exp X) h(\exp Y) \in N^{+} G(\Omega) N^{-}
$$

is a diffeomorphism. Furthermore, $N^{+} G(\Omega) N^{-}$is an open subset in $G\left(T_{\Omega}\right)$ and this decomposition is uniquely determined. Alternate proofs of these facts along with the density of the image in $G\left(T_{\Omega}\right)$ can be found in [1], [2], $[3]$.

\section{The Lie Compression Semigroup of a Symmetric Cone.}

Let $V$ be an euclidean Jordan algebra with the corresponding symmetric cone $\Omega$. Following ideas of [1] or [3], we quickly sketch an alternate route to the group $G:=G\left(T_{\Omega}\right)$ of the previous section. We consider on $V$ the conformal or Kantor-Koecher-Tits group $G:=\mathrm{Cfl}(V)$ of birational maps generated by the translations $t_{v}$ by vectors $v \in V$, by elements of $G(\Omega)$, and by the Jordan inverse $j$ (which is only dense-open defined). Then $G$ is a Lie group of birational maps $\phi$ characterized by the property that the derivative $d \phi(x)$ at any regular point $x$ belongs to $G(\Omega)$, and furthermore is Lie group isomorphic to $G\left(T_{\Omega}\right)$. In this identification $G(\Omega)$ acts in its usual way on $V, N^{+}=t_{V}$ acts as translations, and $N^{-}$acts as $j t_{V} j$. There exists a conformal compactification $\mathcal{M}:=G / P$ with respect to the parabolic subgroup 
$P=G(\Omega) N^{-}$and the embedding of $V$ into $\mathcal{M}$ given by $v \mapsto t_{v} P$ is a dense open equivariant embedding. Thus each transformation of $G$ on $V$ can be extended to a globally defined automorphism of the conformal compactification $\mathcal{M}$. (The manifold $\mathcal{M}$ is diffeomorphic to the Shilov boundary of a bounded symmetric domain $\mathcal{D}$ which is isomorphic to the tube domain $T_{\Omega}$ via a Cayley transform.)

Lemma 7.1. The space $N^{+} G(\Omega) N^{-}$can be characterized by the elements $g \in G\left(T_{\Omega}\right)$ such that $g(0) \in V$.

Proof. We consider the action of $G\left(T_{\Omega}\right)$ on $\mathcal{M}$. If $g(0) \in V$, then $g(0) P=$ $t_{v} P$ for some $v \in V$, and hence $g=t_{v} h \tilde{t}_{w} \in N^{+} G(\Omega) N^{-}$for some $h, \tilde{t}_{w}$.

In the action of $G=G\left(T_{\Omega}\right)$ on $\mathcal{M}$, we consider the compression semigroup of $\Omega \subset \mathcal{M}$

$$
\Gamma_{\Omega}=\left\{g \in G\left(T_{\Omega}\right) \mid g(\Omega) \subset \Omega\right\} .
$$

Since the closure $\tilde{\Omega}$ of $\Omega$ in $\mathcal{M}$ is compact with $\Omega$ as interior, the compression semigroup $\Gamma_{\Omega}$ is a closed semigroup of $G\left(T_{\Omega}\right)$. Using Proposition B.1.3 [10], we have the following.

Proposition 7.2. The interior of $\Gamma_{\Omega}$ is

$$
\Gamma_{\Omega}^{0}=\left\{g \in G\left(T_{\Omega}\right) \mid g(\tilde{\Omega}) \subset \Omega\right\} .
$$

Let $\mathbf{C}=\left\{(u, 0,-v) \in \mathfrak{g}\left(T_{\Omega}\right) \mid u, v \in \bar{\Omega}\right\}$. Then $\mathbf{C}$ is a pointed closed convex cone in $\mathfrak{q}$ with interior

$$
\mathbf{C}^{0}=\left\{(u, 0,-v) \in \mathfrak{g}\left(T_{\Omega}\right) \mid u, v \in \Omega\right\} .
$$

We recall that any element of $G(\Omega)_{0}$, the identity component of $G(\Omega)$, can be written in the form $P(w) k$ for some $w \in V$ and some Jordan algebra automorphism $k$ of $V$, and that the identity component acts transitively on $\Omega$.

Proposition 7.3. For $X \in \mathbf{C}^{0}, \operatorname{ad}_{X}$ is hyperbolic. Hence $\mathbf{C}$ is a hyperbolic cone.

Proof. Let $X=(u, 0,-v) \in \mathbf{C}^{0}$. Choose $h=P(w) k \in G(\Omega)^{0}$ such that $h(e)=u$. Pick $z \in \Omega$ such that $P\left(w^{-1}\right) k(z)=v$. Then since $j P(w) j=$ $P\left(w^{-1}\right)$ (see $\left.[\mathbf{1}]\right), \operatorname{Ad}_{h}(e, 0,-z)=(u, 0,-v)=X$. Now we may take $a \in \Omega$ such that $a^{-4}=z$. Then $A d_{P(a)}\left(a^{-2}, 0,-a^{-2}\right)=(e, 0,-z)$ and $\left(a^{2}, 0,-a^{2}\right) \in$ $\mathfrak{p}$, hence is hyperbolic. Thus $X \in A d_{H}(\mathfrak{p})$ is hyperbolic.

We have now (among other things) established the hypotheses of Theorem 4.1 in our context of Jordan algebras and thus can deduce the following result.

Theorem 7.4. Define $S=G(\Omega) \exp$ C. Then $S$ is a closed semigroup in $G\left(T_{\Omega}\right)$ with $S^{0}=G(\Omega) \exp \mathbf{C}^{0}$ as interior. Furthermore, the tangent wedge of $S$ is $\mathcal{L}(S)=\mathfrak{h}+\mathbf{C}$. 
Now let

$$
\begin{aligned}
& \Gamma^{+}=\left\{t_{x} \in N^{+} \mid x \in \bar{\Omega}\right\}=\exp \bar{\Omega}, \\
& \Gamma^{-}=\left\{\tilde{t}_{-x} \in N^{-} \mid x \in \bar{\Omega}\right\}=\exp \theta(-\bar{\Omega}) .
\end{aligned}
$$

Then it easy to see that $\Gamma^{ \pm}$, and $G(\Omega)$ are closed subsemigroups in $\Gamma_{\Omega}$ and $S$. Hence $\Gamma^{+} G(\Omega) \Gamma^{-} \subset \Gamma_{\Omega} \cap S$.

Lemma 7.5. For $x \in V$, if $x+\Omega \subset V^{-1}$, then $x+\Omega \subset \Omega$. In particular, $x \in \bar{\Omega}$. Conversely if $x \in \bar{\Omega}$, then $x+\Omega \subset \Omega$.

Proof. Since $\Omega$ is open, we can choose $w \in \Omega$ and $t>0$ such that $t x+w \in \Omega$. Then

$$
x+\frac{1}{t} w \in \Omega \cap(x+\Omega) .
$$

This implies that $x+\Omega$ is a connected subset of $V^{-1}$ meeting the symmetric cone $\Omega$. Using the characterization of $\Omega$ in Theorem 6.1 , we conclude that $x+\Omega \subset \Omega$. Hence $x+\bar{\Omega} \subset \bar{\Omega} \Longrightarrow x \in \bar{\Omega}$. Suppose that $x \in \bar{\Omega}$. Then $x+\Omega$ is open in $\bar{\Omega}$ and hence it is a subset of $\Omega$.

Theorem 7.6. For the compression semigroup $\Gamma_{\Omega}$ of the symmetric cone $\Omega$ of a euclidean Jordan algebra,

$$
\Gamma_{\Omega}=N^{+} G(\Omega) N^{-} \cap \Gamma_{\Omega}=\Gamma^{+} G(\Omega) \Gamma^{-},
$$

and this semigroup is equal to the semigroup $S$ of Theorem 7.4.

Proof. It follows from the preceding section, from the semisimplicity of $G$, from Proposition 7.3, and from Theorem 5.3 that $\Gamma^{+} G(\Omega) \Gamma^{-}$is a closed subsemigroup which is equal to the Ol'shanski semigroup $S$.

Obviously $\Gamma^{+} G(\Omega) \Gamma^{-} \subseteq N^{+} G(\Omega) N^{-} \cap \Gamma_{\Omega}$. Conversely, suppose $g=$ $t_{x} \circ h \circ \tilde{t}_{y} \in \Gamma_{\Omega}$. We note that $\tilde{t}_{y}(\Omega)=j\left(y-\Omega^{-1}\right)=j(y-\Omega)$. Because $g(\Omega) \subseteq \Omega \subset V$ and $t_{x} \circ h(V)=V$,

$$
j(y-\Omega)=\tilde{t}_{y}(\Omega)=\left(t_{x} \circ h\right)^{-1} g(\Omega) \subseteq\left(t_{x} \circ h\right)^{-1}(\Omega) \subseteq V .
$$

It follows that $y-\Omega$ and hence $\Omega-y$ is a subset of $V^{-1}$. Hence $-y \in \bar{\Omega}$ from Lemma 7.5. Let $w=-y \in \bar{\Omega}$. Then $w+\Omega \subseteq \Omega$.

Choose $z \in w+\Omega$ and write $z=w+a \in w+\Omega$. Then

$$
2 z=2 w+2 a=w+(w+2 a) \in w+(w+\Omega) \subseteq w+\Omega .
$$

By induction $n z \in w+\Omega$ for all positive integers $n$. Since $(n z)^{-1}=(1 / n) z^{-1}$ and $t_{x} h j(-(w+\Omega)) \subset \Omega$, we conclude that

$$
x+\frac{1}{n} h\left(z^{-1}\right) \in \Omega .
$$

Taking the limit as $n \rightarrow \infty$, we conclude $x \in \bar{\Omega}$. Thus $g \in \Gamma^{+} G(\Omega) \Gamma^{-}$and the second equality is established.

Let $g \in \Gamma_{\Omega}$. Then $g_{n}^{\prime}:=g \circ t_{(1 / n) e} \in \Gamma_{\Omega}$ and $g_{n}^{\prime}(0)=g\left(\frac{1}{n} e\right) \in \Omega$. Hence $g_{n}^{\prime} \in N^{+} G(\Omega) N^{-}$by Lemma 7.1. From the first part of the proof $g_{n}^{\prime}$ must 
be in $\Gamma^{+} G(\Omega) \Gamma^{-}$. As $n \rightarrow \infty$, we obtain $g \in \overline{\Gamma^{+} G(\Omega) \Gamma^{-}}=\Gamma^{+} G(\Omega) \Gamma^{-}$. Since we remarked right after the definition of $\Gamma^{ \pm}$that $\Gamma^{+} G(\Omega) \Gamma^{-} \subseteq \Gamma_{\Omega}$, we conclude that they are equal.

Koufany [12] has established the triple decomposition for the compression semigroup of a symmetric cone using the theory of Jordan pairs and other technical tools from the theory of Jordan algebras. We have preferred to develop the theory in the context of Lie semigroup theory.

Corollary 7.7. Let $S^{0}$ be the interior of the semigroup of $S$. Let $U=$ $\{(u, 0,-e) \mid u \in \Omega\}$. Then

$$
S^{0}=G(\Omega) \exp C^{0}=\Gamma_{0}^{+} G(\Omega) \Gamma_{0}^{-}=G(\Omega)(\exp U) G(\Omega) .
$$

Proof. The first two equalities and the inclusion $G(\Omega)(\exp U) G(\Omega) \subseteq S^{0}$ follow easily from Theorems 5.3 and 7.6. Conversely, if $X=(u, 0,-v) \in C^{0}$, then by definition, $u, v \in \Omega$. Since $G(\Omega)$ acts transitively on $\Omega$, there is $h \in$ $G(\Omega)$ such that $h(e)=v$. It then follows that $\operatorname{Ad}(h)\left(\operatorname{Ad}\left(h^{-1}\right)(u), 0,-e\right)=$ $(u, 0,-v)$, and $\exp X \subset G(\Omega)(\exp U) G(\Omega)$. Hence we have that $G(\Omega) \exp C^{0}$ $\subset G(\Omega)(\exp U) G(\Omega)$.

\section{The Compression Semigroup of the Positive Definite Matrices.}

Let $V:=\operatorname{Sym}(n, \mathbb{R})$ denote the euclidean Jordan algebra of real symmetric matrices equipped with the Jordan product

$$
A \cdot B:=\frac{A B+B A}{2} .
$$

Note that matrix inversion agrees with Jordan inversion in this example. We (densely) define $j(X)=-X^{-1}$. The associated symmetric cone is the cone $\Omega:=\operatorname{Sym}^{+}(n, \mathbb{R})$ consisting of all positive definite symmetric matrices. In this case the group $\operatorname{Sp}(2 n, \mathbb{R})$ is a double covering of the automorphism group of biholomorphisms on the tube domain

$$
T_{\Omega}=\left\{X+i Y \in \operatorname{Sym}(n, \mathbb{C}): Y \in \operatorname{Sym}^{+}(n, \mathbb{R})\right\},
$$

where the action is by fractional transformations. The group $\operatorname{Sp}(2 n, \mathbb{R})$ also acts on $V$ as (densely defined) birational maps of fractional transformations:

$$
X \mapsto(A X+B)(C X+D)^{-1},\left[\begin{array}{cc}
A & B \\
C & D
\end{array}\right] \in \mathrm{Sp}(2 n, \mathbb{R}) .
$$

We define the involution $\tau: \operatorname{Sp}(2 n, \mathbb{R}) \rightarrow \operatorname{Sp}(2 n, \mathbb{R})$ by

$$
\tau\left(\left[\begin{array}{ll}
A & B \\
C & D
\end{array}\right]\right)=\left[\begin{array}{ll}
I & 0 \\
0 & -I
\end{array}\right]\left[\begin{array}{ll}
A & B \\
C & D
\end{array}\right]\left[\begin{array}{ll}
I & 0 \\
0 & -I
\end{array}\right]=\left[\begin{array}{cc}
A & -B \\
-C & D
\end{array}\right] .
$$

The fixed point group $H$ for $\tau$ consists of all block diagonal matrices in $\operatorname{Sp}(2 n, \mathbb{R})$. Further $\dot{\tau}: \mathfrak{g} \rightarrow \mathfrak{g}$ defines a symmetric algebra of Cayley type 
with

$$
\mathfrak{g}=\mathfrak{h}+\mathfrak{q}=\mathfrak{n}^{+}+\mathfrak{h}+\mathfrak{n}^{-}, \text {where } \mathfrak{h}=\left\{\left[\begin{array}{cc}
A & 0 \\
0 & -A^{t}
\end{array}\right]: A \in \operatorname{gl}(n, \mathbb{R})\right\},
$$

and

$$
\mathfrak{n}^{-}=\left\{\left[\begin{array}{ll}
0 & B \\
0 & 0
\end{array}\right]: B=B^{t}\right\}, \mathfrak{n}^{+}=\left\{\left[\begin{array}{ll}
0 & 0 \\
B & 0
\end{array}\right]: B=B^{t}\right\} .
$$

The exponential images $N^{ \pm}$of $\mathfrak{n}^{ \pm}$resp. consist of those block unipotent lower resp. upper triangular matrices with $B=B^{t}$. Note that $N^{-}$acts on $V$ by translation $t_{B}$ by the upper right entry $B$, and a quick calculation establishes that $N^{+}$acts on $V$ by $\tilde{t}_{-B}=j \circ t_{-B} \circ j$, where $B$ is now the lower left entry.

The compression semigroup of the symmetric cone $\Gamma:=\Gamma_{\Omega}$ consists of all $g \in \operatorname{Sp}(2 n, \mathbb{R})$ which are globally defined on $\Omega$ and satisfy $g \cdot \Omega \subseteq \Omega$. If $\bar{\Omega}$ is embedded in $\mathfrak{n}^{-}$as the upper right entry and in $\mathfrak{n}^{+}$by putting its negative as the lower left entry, then the resulting cones $\mathbf{C}^{-}$and $\mathbf{C}^{+}$are those that we have seen arising from Section 4 forward. It then follows from the preceding section that $\Gamma=\exp \left(\mathbf{C}^{-}\right) H \exp (\mathbf{C})$, where the triple decomposition is uniquely determined.

The compression semigroup $\Gamma$ has appeared previously in Wojtkowski's study of the entropy of a system of hard spheres and in the stochastic calculus in the work of Bougerol [4]. Bougerol actually considered the semigroup

$$
S:=\left\{\left[\begin{array}{cc}
A & B \\
C & D
\end{array}\right] \in \operatorname{Sp}(2 n, \mathbb{R}): D\right. \text { is invertible, }
$$

$$
\left.C D^{t}, D^{t} B \text { are positive semidefinite }\right\} \text {, }
$$

but in the introduction of his work Koufany [12] remarks that $S$ is easily shown to be equal to $\Gamma$. Using the invertibility of $D$ for elements of the semigroup $\Gamma$ (this condition is also derived by Wojtkowski) we obtain directly the triple factorization in $\Gamma$. Indeed we can characterize and give explicitly the triple factorization of Theorem 5.2.

Theorem 8.1. An element $g=\left[\begin{array}{cc}A & B \\ C & D\end{array}\right] \in \operatorname{Sp}(2 n, \mathbb{R})$ can be written (uniquely) as a triple product in $\mathrm{N}^{-} \mathrm{HN}^{+}$if and only if $D$ is invertible. In this case the (unique) factorization is given by

$$
\left[\begin{array}{ll}
A & B \\
C & D
\end{array}\right]=\left[\begin{array}{ll}
I & B D^{-1} \\
0 & I
\end{array}\right]\left[\begin{array}{ll}
\left(D^{-1}\right)^{t} & 0 \\
0 & D
\end{array}\right]\left[\begin{array}{ll}
I & 0 \\
D^{-1} C & I
\end{array}\right] .
$$

The factors are all in $\Gamma$ if and only if $g \in \Gamma$.

Proof. If $g \in N^{-} \mathrm{HN}^{+}$, then one verifies by direct multiplication that $D$ is the same as the bottom right entry in the $H$-coordinate, and the latter 
must be invertible. Conversely if $D$ is invertible, then the above factorization works. (One must use the fact that $B^{t} D$ is symmetric and that $A^{t} D-C^{t} B=$ $I$ since the matrix is in $\operatorname{Sp}(2 n, \mathbb{R})$ to establish that the upper left entry in the triple product is A.) The uniqueness follows from Theorem 5.2. Since we have already established that the factorization holds in $\Gamma$ and since the factorization is unique, the last assertion follows.

\section{References}

[1] W. Bertram, Algebraic structures of Makarevic spaces, I, II, Preprint, Institut MittagLefffler 1996; to appear in 'Transformation Groups'.

[2] _ On some causal and conformal groups, J. Lie Theory, 6 (1996), 215-244.

[3] _ Un théorème de Liouville pour les algèbres de Jordan, Bull. Soc. Math. France, 124 (1996), 299-327.

[4] Ph. Bougerol, Kalman filtering with random coefficients and contractions, SIAM J. Control and Optimization, 31 (1993), 942-959.

[5] J. Faraut, Espaces symétriques ordonnés et algèbres de Volterra, J. Math. Soc. Japan, 43 (1991), 133-147.

[6] J. Faraut and A. Koranyi, Analysis on Symmetric Cones, Oxford Press, Oxford, 1994.

[7] S. Helgason, Differential Geometry, Lie Groups, and Symmetric Spaces, Acad. Press, Orlando, 1978.

[8] J. Hilgert, K.H. Hofmann and J.D. Lawson, Lie Groups, Convex Cones, and Semigroups, Oxford Press, Oxford, 1989.

[9] J. Hilgert and K.-H. Neeb, Basic Theory of Lie Semigroups and Applications, Springer Lecture Notes in Mathematics, 1552, Springer, 1993.

[10] J. Hilgert and G. Ólafsson, Causal Symmetric Spaces, Academic Press, San Diego, 1997.

[11] K.H. Hofmann, J.D. Lawson and E.B. Vinberg, Editors, Semigroups in Algebra, Geometry and Analysis, de Gruyter, Berlin, 1995.

[12] K. Koufany, Semi-groupe de Lie associé a un cone symmétrique, Ann. Inst. Fourier, Grenoble, 45 (1995), 1-29.

[13] B. Krötz and K.-H. Neeb, On hyperbolic cones and mixed symmetric spaces, Journal of Lie Theory, 6 (1996), 69-146.

[14] J.D. Lawson, Ordered manifolds, invariant cone fields, and semigroups, Forum Math., 1 (1989), 273-308.

[15] _ Polar and Ol'shanski decompositions, J. Reine Angew. Math., 448 (1994), 191-219.

[16] Semigroups of Ol'shanski type, Semigroups in Algebra, Geometry and Analysis, de Gruyter, Berlin, 1994.

[17] D. Mittenhuber and K.-H. Neeb, On the exponential function on ordered manifolds with affine connections, Math. Z., 218 (1995), 1-23.

[18] G.I. Ol'shanski, Convex cones in symmetric Lie algebras, Lie semigroups, and invariant causal structures on pseudo-Riemannian symmetric spaces, Sov. Math. Dokl., 26 (1982), 97-101. 
[19] _ Invariant cones in Lie algebras, Lie semigroups, and the holomorphic discrete series, Funct. Anal. and Appl., 15 (1982), 275-285.

[20] M. Wojtkowski, Measure theoretic entropy of the system of hard spheres, Ergod. Th. and Dynam. Sys., 8 (1986), 133-153.

Received August 19, 1998 and revised June 7, 1999. The authors gratefully acknowledge the support of the National Science Foundation and TGRC-KOSEF respectively.

Louisiana State University

Baton Rouge, LA 70803-4918

E-mail address: lawson@math.lsu.edu

KYUngPoOK NATIONAL UnIVERSITY

TAEgu 702-701, Korea

E-mail address: ylim@kyungpook.ac.kr 